\title{
Can integrated health services delivery have an impact on hypertension management? A cross-sectional study in two cities of China
}

Haitao Li ${ }^{1}$, Ying Sun ${ }^{2}$ and Dongfu Qian ${ }^{3^{*}}$

\begin{abstract}
Background: Policy makers require information regarding performance of different primary care delivery models in managing hypertension, which can be helpful for better hypertension management. This study aims to compare continuity of care among hypertensive patients between Direct Management (DM) Model of community health centers (CHCs) in Wuhan and Loose Collaboration (LC) Model in Nanjing.

Methods: A cross-sectional questionnaire survey was conducted. Four CHCs in each city were randomly selected as study settings. 386 patients in Nanjing and 396 in Wuhan completed face-to-face interview surveys and were included in the final analysis. The relational continuity and coordination continuity (including both information continuity and management continuity) were measured and analyzed. Binary or multinomial logistic regression models were used for comparison between the two cities.

Results: Participants from Nanjing had better relational continuity with primary care providers as compared with those from Wuhan, including more likely to be familiar with a $\mathrm{CHC}$ physician $(\mathrm{OR}=2.762 ; 95 \% \mathrm{Cl}: 1.878$ to 4.061$)$, taken care of by the same $\mathrm{CHC}$ physician ( $\mathrm{OR}=1.846 ; 95 \% \mathrm{Cl}: 1.262$ to 2.700$)$, and known well by a $\mathrm{CHC}$ physician $(\mathrm{OR}=1.762 ; 95 \% \mathrm{Cl}$ : 1.206 to 2.572). Multinomial logistic regression analyses showed there were significant differences between the two cities in reported frequency of communications between hospital and CHC physicians ( $P=0.001$ ), whether hospital and CHC physicians gave same treatment suggestions $(P=0.016)$, as well as how treatment strategy was formulated $(P<0$. 001). Participants in Wuhan were less likely than those in Nanjing to consider there was continuum regarding health services provided by hospital and CHC physicians ( $\mathrm{OR}=3.932 ; 95 \% \mathrm{Cl}$ : 2.394 to 6.459$)$.
\end{abstract}

Conclusions: Our study shows that continuity of care is better for LC Model in Nanjing than DM Model in Wuhan. Our study suggests there is room for improvement regarding relational and information continuity in both cities.

Keywords: Primary care, Continuity of care, Hypertension, High blood pressure

\section{Background}

Hypertension is an important public health problem faced by policy makers worldwide and China. It is reported that one in three adults had hypertension throughout the world in 2012 [1]. It is projected that there will be 1.56 billion adults living with hypertension in 2025 [2]. In China, prevalence of hypertension has

\footnotetext{
* Correspondence: dqian@njmu.edu.cn

${ }^{3}$ School of Health Policy and Management, Nanjing Medical University, Hanzhong Road 140, Nanjing 210029, Jiangsu Province, China

Full list of author information is available at the end of the article
}

increased sharply during the past several decades - escalated from about $8 \%$ in 1979 [3] to $34 \%$ in 2010 [4]. World Health Organization (WHO) recognizes hypertension as one of the most important causes of premature death. Estimates suggest that hypertension causes about 7.5 million deaths annually, accounting for $12.8 \%$ of total deaths in the world [5]. In China, about $50 \%$ of deaths are attributable to pre-hypertension and hypertension, which is recognized to be the leading cause of premature death [6]. 
Hypertension control is thus important to reduce the risks of diseases with hypertension as a key risk factor. However, hypertension is sub-optimally controlled throughout the world including China. A study with a nationally representative sample of US population showed that hypertension control rate was $53 \%$ in 2010 [7]. In Canada, approximately $66 \%$ of hypertensive patients had their blood pressure under control [8]. The Chinese national hypertension survey in 2002 showed that only 4\% of patients with hypertension met blood pressure control target [9]. The WHO Report 2008 has emphasized the relevance of primary care in coping with the increased challenges of chronic diseases including hypertension. The New Healthcare Reform Plan launched by the Chinese government in 2009 highlighted the key role of primary care in managing hypertension [10]. The WHO Report 2013 outlines primary care programs for any country initiative to address hypertension.

In China, primary care, named as community health services, is usually provided by community health centers (CHCs) in urban areas and township hospitals in rural areas. Six-integrated health services are designed to be provided by primary care facilities including medical care, preventive care, rehabilitation, health education and promotion, chronic disease management and technical support for family planning [11]. Health personnel usually consist of general practitioners, Traditional Chinese Medicine doctors, nurses and public health doctors etc [12]. Usually, there is a loose collaboration between hospitals and CHCs, namely Loose Collaboration Model (LC Model). CHCs in Nanjing City, Jiangsu Province are LC Models, which are separate and independent organizations from hospitals. Hospitals just provide technical guidance to $\mathrm{CHCs}$, such as training sessions on specific topics. International experience has shown that close cooperation between hospitals and primary care facilities can provide patients with a seamless and interconnected healthcare, which is widely recognized to be able to improve quality of care and lower healthcare costs $[13,14]$. Primary care reform in China has targeted increased service integration and multidisciplinary coordination. One model of care for integrated health services delivery has emerged, i.e., Direct Management Model (DM Model). Wuhan City, Hubei Province is a pioneer in exploring and developing the DM Model in China. In this model, CHCs are similar to departments of a hospital. Hospital is responsible for the overall operation of its affiliated $\mathrm{CHCs}$, playing an important financial and administrative role. For example, the personnel of $\mathrm{CHCs}$ are managed by the hospital.

Continuity of care is defined by the Institute of Medicine as one key attribute of primary care [15]. Continuity of care refers to both relational continuity between patient and primary care provider and care continuity between primary care provider and specialist such that a patient seamlessly experiences care across different providers (coordination) [16]. The longitudinal relationship ideally leads to a bond between physicians and patients, characterized by trust and a sense of responsibility [17]. Coordination continuity consists of information continuity which refers to communicating timely clinical and life information about a patient's condition between providers, and management continuity which means coordinating medical services and care pathways between multiple providers and settings in response to ongoing care needs [18]. Continuity is essential for the care necessary for chronically ill patients, since cultivating a relationship with a single provider builds up knowledge of a patient's preferences and can help integrate a patient's care between different providers.

Primary care reform in China has targeted increased service integration and multidisciplinary coordination. DM Model is the latest form of CHC-hospital collaboration. Policy makers require information regarding performance of DM Model in managing hypertension, which can be helpful for better management of hypertension and in ensuring better development of DM Model. To our knowledge, the information is scarce with respect to performance of DM Model in managing hypertension. This study aimed to compare performance of DM Model in Wuhan with LC Model in Nanjing in managing hypertension, which is measured by continuity of care.

\section{Methods}

\section{Participants and procedures}

A cross-sectional study was conducted in Wuhan and Nanjing in 2012. Nanjing is the capital of Jiangsu Province and the second largest city in eastern China, while Wuhan is the capital of Hubei Province and the most populous city in central China. Both Nanjing and Wuhan hold sub-provincial administrative status. In 2013, GDP per capita was RMB 98 thousand (US\$16 thousand) for Nanjing [19], and RMB 89 thousand (US\$14 thousand) for Wuhan [20]. Both cities are leading primary care development in their own $\mathrm{CHC}$ hospital collaboration models. A multistage sampling method was used to select $\mathrm{CHCs}$ as study settings. In the first stage, one typical tertiary general hospital that has developed cooperation relations with local $\mathrm{CHCs}$ was selected in each city using a representative sampling. In the second stage, deployed simple random sampling methods, four $\mathrm{CHCs}$ in each city, which collaborated with the recruited typical hospitals, were selected randomly. At last, a total of $8 \mathrm{CHCs}$ were selected as study settings. In this study, all selected $\mathrm{CHCs}$ and hospitals are public health institutions and owned by the government.

The sampling frame was CHC users' hypertensive population based. It was shown that a maximum sample 
size of 300 per group was needed to generate a $95 \%$ confidence interval and $90 \%$ statistical power [21]. Inclusion criteria of participants were: 1) aged between 18 and 80 years; 2) with confirmed diagnosis of hypertension; 3 ) living in the catchment area of $\mathrm{CHC} \geq 1$ year; 4) had experience of both specialty care and primary care utilization during the past 1 year period. We excluded patients who had severe hypertension complications and terminal illnesses. A systematic sampling design was adopted to ensure that only patients coming to the $\mathrm{CHC}$ for primary care during the survey period were invited for inclusion. A hundred eligible patients were approached in each included $\mathrm{CHC}$. Extensively trained interviewers performed on-site based face-to-face interview surveys. Participants were assured of anonymity and confidentiality of the survey, and verbal informed consent was obtained before the surveys were commenced. Among the approached patients, 386 in Nanjing and 396 in Wuhan completed face-to-face interview surveys with a response rate of 96.5 and $99.0 \%$ respectively. The study was approved by the Research Ethics Committee of Nanjing Medical University.

\section{Key measures}

We conceptualized three dimensions of continuity of care (i.e., relational continuity, information continuity and management continuity) in Haggerty's model of continuity of care. To understand the longitudinal relationship between primary care provider and patient (relational continuity), we asked three questions. 1) Is there any $\mathrm{CHC}$ physician that you are familiar with? 2) When you go to $\mathrm{CHC}$, are you taken care of by the same physician each time? 3) Does the physician in CHC knows you well, including your most important health problems, your complete medical history, and medications you are taken?

As for coordination continuity, we asked several questions to measure information continuity. 1) How often do hospital-based physicians communicate with primary care providers (CHC physicians)? 2) How do you think the communication results between hospital-based physicians and primary care providers (CHC physicians)? 3 ) Does your specialist give the same treatment suggestions as that of your primary care providers ( $\mathrm{CHC}$ physicians)? 4) Who formulated treatment strategy for you? As for management continuity, it was measured by one question: 1) Do you think there is continuum regarding health services provided by hospital-based physicians and primary care providers ( $\mathrm{CHC}$ physicians)?

According to the framework of Behavioral Model of Health Services Utilization, individual factors that may influence health care use were collected. Individual factors consist of predisposing factors (including gender, age, marital status and education level), enabling factors (including monthly household income), as well as need factors (including year of hypertension since diagnosis, complications, self-reported overall health status and blood pressure measures). We grouped marital status into two categories, those single (including not married, separated, widowed and divorced), and those currently married or cohabited. Education level was collapsed into four categories, i.e., primary school and below, middle school, high school and equivalent, as well as 3-year college and above. According to mean monthly household income in 2012 (RMB3000 for Nanjing [19], and RMB2400 for Wuhan [20]), the participants were grouped into two economic levels- below or above mean monthly household income. Hypertension-related complications included cerebrovascular disease, heart disease and kidney disease. Blood pressure (BP) was measured by trained interviewers according to Chinese Hypertension Management Guidelines. BP control was determined by whether a participant met BP control target level, i.e., <140/ $90 \mathrm{mmHg}$.

\section{Statistical analysis}

The socio-demographic characteristics and hypertensionrelated information between the participants from the two cities were compared by using Chi-square tests (or independent two-sample t-tests where appropriate). We firstly employed Chi-square tests to compare participants' reported experiences of continuity of care between the two cities. Then, binary or multinomial logistic regression models were used for comparison between the two cities by controlling for participants' gender, age, education level, marital status, monthly household income, overall health status, year of hypertension since diagnosis and hypertension-related complications. All data were tested to establish if they violated assumptions of the multinomial logistic regression; though this type of regression does not assume normality, linearity, or homoscedasticity. Adjusted odds ratio (OR) with 95\% confidence interval (CI) was reported where Wuhan was regarded as the reference group. The likelihood ratio test statistic was used to test the fit of model. For all tests conducted in the study, a $P$ value of less than 0.05 was adopted as the statistically significant level. All analyses were performed by using SPSS19.0.

\section{Results}

Compared with those from Wuhan, participants from Nanjing were more likely to be male ( 51.3 vs $43.2 \%$, $P=0.026)$. In both cities, the majority of participants tended to be aged more than 60 years old (69.8 vs $87.4 \%$ ) and married or cohabited (91.2 vs $89.4 \%$ ). Participants from Nanjing tended to possess a higher education level when compared with those from Wuhan $(P<0.001)$. More participants' monthly household income 
in Nanjing than in Wuhan was higher than the mean level in each city $(P<0.001)$, while more than one third participants from Nanjing rejected to report their income. Participants' overall health status in Nanjing was poorer than those in Wuhan $(P=0.009)$. When compared with those from Wuhan, participants from Nanjing were less likely to have hypertension-related complications (32.8 vs $48.3 \%, P<0.001)$, while more likely to have their BP under control (38.9 vs $28.9 \%, P=0.003$ ) (Table 1 ).

Significant differences between the two cities were identified regarding relational continuity. After adjusting for socio-demographic characteristics and hypertensionrelated factors, participants from Nanjing tended to report that there was a $\mathrm{CHC}$ physician that they were

Table 1 Characteristics of the participants by city

\begin{tabular}{|c|c|c|c|}
\hline Characteristics & $\begin{array}{l}\text { Nanjing } \\
\mathrm{N}(\%)\end{array}$ & $\begin{array}{l}\text { Wuhan } \\
\mathrm{N}(\%)\end{array}$ & $P$ value \\
\hline \multicolumn{4}{|l|}{ Socio-demographic } \\
\hline Gender & & & 0.026 \\
\hline Male & 198(51.3) & $171(43.2)$ & \\
\hline Female & 188(48.7) & $225(56.8)$ & \\
\hline Age & & & $<0.001$ \\
\hline$<60$ & 118(30.2) & $50(12.6)$ & \\
\hline$>=60$ & 273(69.8) & $348(87.4)$ & \\
\hline Marital status & & & 0.464 \\
\hline Single & $33(8.8)$ & $41(10.6)$ & \\
\hline Married or cohabited & $340(91.2)$ & $347(89.4)$ & \\
\hline Education & & & $<0.001$ \\
\hline Primary school and below & $71(18.7)$ & 123(31.1) & \\
\hline Middle school & $95(25.1)$ & 148(37.4) & \\
\hline High school and equivalent & 107(28.2) & $87(22.0)$ & \\
\hline 3-year college and above & $106(28.0)$ & 38(9.6) & \\
\hline Monthly household income & & & $<0.001$ \\
\hline Low & $45(11.5)$ & $110(27.6)$ & \\
\hline High & 195(49.9) & $274(68.8)$ & \\
\hline Rejected & 151(38.6) & $14(3.5)$ & \\
\hline \multicolumn{4}{|l|}{ Disease-related } \\
\hline Year of hypertension (mean, SE) & $10.48(0.51)$ & $11.91(0.50)$ & 0.046 \\
\hline Health status & & & 0.009 \\
\hline Good & 153(40.2) & 197(49.6) & \\
\hline Fair or poor & $228(59.8)$ & $200(50.4)$ & \\
\hline Complications & & & $<0.001$ \\
\hline Yes & 124(32.8) & 185(48.3) & \\
\hline No & $254(67.2)$ & 198(51.7) & \\
\hline BP control & & & 0.003 \\
\hline Yes & 152(38.9) & 115(28.9) & \\
\hline No & 239(61.1) & $283(71.1)$ & \\
\hline
\end{tabular}

SE standard error familiar with $(56.4 \%$ vs $31.5 \%$; OR $=2.762$; $95 \% \mathrm{CI}$ : 1.878 to 4.061); they were taken care of by the same $\mathrm{CHC}$ physician each time ( $47.1 \%$ vs $33.4 \%$; OR $=1.846$; $95 \%$ CI: 1.262 to 2.700); and, $\mathrm{CHC}$ physician knew them well including their most important problems and complete medical history $(58.3 \%$ vs $46.8 \%$; OR $=1.762$; $95 \%$ CI: 1.206 to 2.572$)$ when compared with those from Wuhan (Table 2).

As for information continuity, multinomial logistic regression analyses showed that there were significant differences between the two cities in reported frequency of communications between hospital and $\mathrm{CHC}$ physicians, whether hospital and $\mathrm{CHC}$ physicians gave same treatment suggestions, as well as how treatment strategy was formulated (Table 3). Compared with those in Wuhan, participants in Nanjing tended to report that communications between hospital and $\mathrm{CHC}$ physicians were fair (19.1 vs $8.7 \%$; OR $=3.019$; $95 \%$ CI: 1.723 to 5.288 ) or few ( 18.9 vs $15.3 \%$; OR $=1.695$; $95 \%$ CI: 1.020 to 2.817 ). More participants in Nanjing than in Wuhan reported that suggestions provided by hospital physicians were the same as that of $\mathrm{CHC}$ physicians (33.1 vs $23.0 \%$; OR = 1.869; 95\%CI: 1.211 to 2.884 ). Participants in Wuhan were less likely to report that their treatment strategy was formulated by $\mathrm{CHC}$ physicians (50.5 vs $32.5 \%$; $\mathrm{OR}=8.553 ; 95 \% \mathrm{CI}: 4.247$ to 17.229$)$ or by $\mathrm{CHC}$ and hospital physicians together (8.8 vs $1.0 \%$; OR $=32.825$; 95\%CI: 8.355 to 128.963 ) when compared with those in Nanjing (Table 2).

When it comes to management continuity, Table 2 shows that participants in Wuhan were less likely than those in Nanjing to consider there was continuum regarding health services provided by hospital and $\mathrm{CHC}$ physicians $(72.7 \%$ vs $41.5 \%$; OR $=3.932$; $95 \% \mathrm{CI}$ : 2.394 to 6.459), after adjusting for participant socio-demographic characteristics and hypertension-related factors by using binary logistic regression models (Table 2).

\section{Discussion}

Our study found that participants in Nanjing were more likely to have a $\mathrm{CHC}$ physician they were familiar with, be taken care of by the same $\mathrm{CHC}$ physician for each $\mathrm{CHC}$ visit, and have a $\mathrm{CHC}$ physician knowing their disease history. Although there were more participants in Wuhan than in Nanjing did not know whether there was any communication between hospital and $\mathrm{CHC}$ physicians, the participants in Nanjing tended to consider that the communications between hospital and $\mathrm{CHC}$ physicians were fair or few. Similarly, more participants in Nanjing than in Wuhan perceived that the suggestions between hospital and $\mathrm{CHC}$ physicians were same; though more participants in Wuhan did not know whether they had same suggestions. There were more participants reported that their treatment strategy was formulated by $\mathrm{CHC}$ physicians together with hospital 
Table 2 Patient reported experiences of continuity of care by city

\begin{tabular}{|c|c|c|c|c|}
\hline Variables & Nanjing & Wuhan & $P$ value ${ }^{a}$ & OR $(95 \% \mathrm{Cl})^{\mathrm{b}}$ \\
\hline \multicolumn{5}{|l|}{ Relational continuity } \\
\hline CHC Physician you are familiar with & & & $<0.001$ & $2.762(1.878,4.061)^{* * *}$ \\
\hline Yes & 212(56.4) & 123(31.5) & & \\
\hline No & 164(43.6) & $267(68.5)$ & & \\
\hline Same CHC physician every time & & & $<0.001$ & $1.846(1.262,2.700)^{* *}$ \\
\hline Yes & $179(47.1)$ & 132(33.4) & & \\
\hline No & 201(52.9) & $263(66.6)$ & & \\
\hline CHC physician knowing your disease history & & & 0.002 & $1.762(1.206,2.572)^{* *}$ \\
\hline Yes & $214(58.3)$ & 182(46.8) & & \\
\hline No & 153(41.7) & 207(53.2) & & \\
\hline \multicolumn{5}{|l|}{ Information continuity } \\
\hline Frequency of communications between $\mathrm{CHC}$ and hospital physicians & & & $<0.001$ & \\
\hline Many & $26(7.0)$ & $18(4.8)$ & & $1.460(0.637,1.024)$ \\
\hline Fair & $71(19.1)$ & $33(8.7)$ & & $3.019(1.723,5.288)^{* * *}$ \\
\hline Few & $70(18.9)$ & $58(15.3)$ & & $1.695(1.020,2.817)^{*}$ \\
\hline Do not know & 204(55.0) & 269(71.2) & & Ref \\
\hline Communication results & & & $<0.001$ & \\
\hline Good & $33(10.2)$ & 10(3.6) & & $2.173(0.848,5.567)$ \\
\hline Fair & 209(64.1) & 119(53.1) & & $1.664(1.019,2.718)^{* *}$ \\
\hline Poor & $84(25.8)$ & $95(42.4)$ & & Ref \\
\hline Whether hospital and CHC physicians have same suggestions & & & 0.001 & \\
\hline Yes & 119(33.1) & $83(23.0)$ & & $1.869(1.211,2.884)^{* *}$ \\
\hline No & $26(7.2)$ & $15(4.2)$ & & $1.543(0.666,3.578)$ \\
\hline Do not know & 215(59.7) & 263(72.9) & & Ref \\
\hline How your treatment strategy was formulated & & & $<0.001$ & \\
\hline CHC physician & $190(50.5)$ & $126(32.5)$ & & $8.553(4.247,17.229)^{* * *}$ \\
\hline Hospital physician & 138(36.7) & 157(40.5) & & $4.495(2.243,9.009)^{* * *}$ \\
\hline CHC physician together with hospital physician & $33(8.8)$ & $4(1.0)$ & & $32.825(8.355,128.963)^{* * *}$ \\
\hline No fixed strategy & $15(4.0)$ & $101(26.0)$ & & Ref. \\
\hline \multicolumn{5}{|l|}{ Management continuity } \\
\hline Total continuum between hospital- and CHC- physician provided services & & & $<0.001$ & $3.932(2.394,6.459)^{* * *}$ \\
\hline Yes & $232(72.7)$ & $78(41.5)$ & & \\
\hline No & $87(27.3)$ & $110(58.5)$ & & \\
\hline
\end{tabular}

OR odds ratio, $\mathrm{CHC}$ community health center, Ref reference group

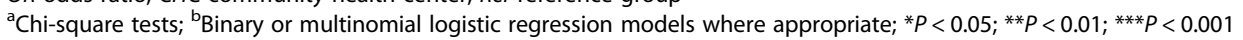

Table 3 Results from multinomial logistic regression likelihood ratio tests showing the differences between Nanjing and Wuhan

\begin{tabular}{|c|c|c|c|c|}
\hline \multirow[t]{2}{*}{ Dependent variables } & \multirow[t]{2}{*}{ Model fitting criteria } & \multicolumn{3}{|c|}{ Likelihood ratio tests } \\
\hline & & Chi-square & df & Sig. \\
\hline Frequency of communications between $\mathrm{CHC}$ and hospital physicians & 1243.14 & 16.746 & 3 & 0.001 \\
\hline Communication results & 765.501 & 5.206 & 2 & 0.074 \\
\hline Whether hospital and CHC physicians have same suggestions & 924.948 & 8.313 & 2 & 0.016 \\
\hline How your treatment strategy was formulated & 1353.31 & 56.469 & 3 & $<0.001$ \\
\hline
\end{tabular}


physicians, although the percentages were low for both cities. In general, management continuity was perceived better by the participants in Nanjing than those in Wuhan.

Limitations of the study should be addressed. Firstly, general applicability of study findings may be limited. For one thing, the study findings cannot be extended to other cities with similar $\mathrm{CHC}$ models as structure of $\mathrm{CHCs}$ with same models may be different in different cities; for another, information were reported by $\mathrm{CHC}$ users and cannot be generalized to hypertensive population in general. Secondly, the representativeness of this study is limited. The representative sampling methods may introduce sampling bias. Moreover, the participants were not selected by using strict random sampling methods. Thirdly, patient-reported information may be subject to recall bias. Fourthly, the items used to measure continuity of care in our study may introduce bias. Further studies are warranted to compare continuity of care between LC Models and DM Models comprehensively. Last but not the least, cross-sectional nature of the current study warrants further investigations to establish causal inferences.

Results showed that participants in Nanjing had better experiences of relational continuity of primary care when compared with those in Wuhan. The differences in health insurance schemes between the two cities may contribute to the differences in relational continuity reported by the participants in the two cities. In Nanjing, reimbursement rate of charges for $\mathrm{CHC}$ services is about $10 \%$ higher than that for hospital services, while in Wuhan that difference is only about $5 \%$ which is lower than that of Nanjing. Financially, $\mathrm{CHC}$ provided primary care may be more attractive in Nanjing than in Wuhan. In other words, patients in Nanjing are more likely to seek primary care than those in Wuhan. Therefore, the participants in Nanjing were more likely to have a $\mathrm{CHC}$ physician they were familiar with. The implementation of First Contact Care Scheme may help to explain this observation too. In Nanjing, it is necessary for patients covered by the Basic Medical Insurance for Urban Residents to obtain referrals from their $\mathrm{CHC}$ physicians for reimbursement of charges of hospital services. The gate-keeping role of $\mathrm{CHC}$ physicians may lead to their knowing more about patients' complete medical history and important health problems. Another possible explanation of this observation is the number of $\mathrm{CHC}$ physicians. Previous studies have shown that smaller practices had better relational continuity [22, 23]. Our study showed that average number of $\mathrm{CHC}$ physicians in Nanjing was smaller than that in Wuhan, which suggests that $\mathrm{CHC}$ physicians in Wuhan have greater sharing of clinical duties resulting in a loss of continuity. Both cities should strengthen relational continuity although it was better in Nanjing than in Wuhan. Enhanced relational continuity can improve hypertensive patients' compliance with medications and follow-up appointments [24].

Results showed that the information continuity was poor in both Wuhan and Nanjing. It was found that the majority of participants in Wuhan did not know the communications between $\mathrm{CHC}$ - and hospital- physicians, while the communications between $\mathrm{CHC}$ - and hospital- physicians were perceived few and poor by the participants in Nanjing although the awareness rate was higher than that in Wuhan. Another item in our study also showed that less than $10 \%$ of treatment strategies were formulated by $\mathrm{CHC}$ - and hospital- physicians together. The poor information sharing system between $\mathrm{CHCs}$ and hospitals may be one possible explanation of this finding. A study by Yang et al. indicated that information sharing system between $\mathrm{CHCs}$ and hospitals in Wuhan has not been well established [25]. It was shown that there was a fragmentation of information systems between CHCs and hospitals in Nanjing [26]. The information sharing system can assist with access to hypertensive patients' medical records leading to improved recognition of hypertensive patients' problems and therapies [27], which are important for better hypertension management. Strategies like adoption of reciprocal referral system, a shift from fee-for-service to global payment, performance-based payment for care providers, and integrated information system may work together to enhance not only relational continuity but also information continuity of care to address the needs of hypertensive patients $[28,29]$.

This study is the first to compare performance of LC Model and DM Model in managing hypertension as measured by continuity of care. This study adds to the evidence suggesting that continuity of care is better for LC Model than for DM Model. Current literature showed mixed views regarding relationship between $\mathrm{CHC}$ models and quality of care. Both CHCs of LC Models and DM Models are publicly owned, but CHCs of DM Models are managed and operated by collaborated hospitals. These hospitals have to rely on profit-generating services to survive financially which influence their managed $\mathrm{CHCs}^{\prime}$ operation or development mode and lead to more profitdriven nature. Publicly owned $\mathrm{CHCs}$ are shown to provide a higher quality of care because they have stronger and better policy implementation [30], such as First Contact Care Scheme and dual-referral system. However, some other studies showed that quality of care offered by forprofit primary care facilities was better than publicly owned ones [31, 32]. The conflicting findings among different studies suggest that performance of $\mathrm{CHCs}$ depends more on the process of hypertension management rather than $\mathrm{CHC}$-hospital collaboration models [11]. 


\section{Conclusions}

Our study found that hypertensive patients in Nanjing had better relational continuity with $\mathrm{CHC}$ physicians than those in Wuhan. Information continuity was found poor in both Nanjing and Wuhan. Our study suggests that there is room for improvement regarding relational continuity and information continuity in both cities.

\section{Abbreviations}

BP: Blood pressure; CHCs: Community health centers; Cl: Confidence interval; DM Model: Direct management model; LC Model: Loose collaboration model; OR: Odds ratio; RMB: Ren Min Bi; WHO: World Health Organization

\section{Acknowledgements}

We would like to thank the National Natural Science Fund of China (grant number: 71173118) and the China Medical Board (grant number: 11-071) to support the study financially. However, the funding bodies played no roles in study design, collection and analysis of data, drafting and submitting of the manuscript. The authors also thank the local staff of Nanjing and Wuhan in coordination of the study. The students who participated in data collection are also acknowledged.

\section{Funding}

This work was supported by the National Natural Science Fund of China (grant number: 71173118) and the China Medical Board (grant number: 11-071).

\section{Availability of data and materials}

All relevant data were presented in the manuscript.

\section{Authors' contributions}

DFQ conceived of the study, and took part in its design. YS participated in data analysis and helped to draft the manuscript. HTL and DFQ drafted the manuscript and were responsible for data interpretation. All authors read and approved the final manuscript.

\section{Competing interests}

The authors declare that they have no competing interests.

\section{Consent for publication}

All authors have read and agreed to publish the manuscript on the International Journal for Equity in Health.

\section{Ethics approval and consent to participate}

The study was approved by the Research Ethics Committee of Nanjing Medical University. Participants were assured of anonymity and confidentiality of the survey, and verbal informed consent was obtained before the surveys were commenced.

\section{Author details}

${ }^{1}$ School of Medicine, Shenzhen University, Shenzhen, China. ${ }^{2}$ The Affiliated Hospital of Qingdao University, Qingdao, China. ${ }^{3}$ School of Health Policy and Management, Nanjing Medical University, Hanzhong Road 140, Nanjing 210029, Jiangsu Province, China.

Received: 15 June 2016 Accepted: 21 November 2016 Published online: 30 November 2016

\section{References}

1. WORLD HEALTH ORGANIZATION. World health stastics 2012. 2012

2. Kearney PM, Whelton M, Reynolds K, Muntner P, Whelton PK, He J. Global burden of hypertension: analysis of worldwide data. Lancet. 2005:365:217-23.

3. Zhou H.Study on evaluation index system and method for comprehensive prevention and control community based for hypertension. Wuhan: Huazhong Technology University; 2004

4. Chinese Center for Disease Control and Prevention. Report on chronic disease riak factor surveilance in China 2010. Beijing: Military Medical Science Press; 2012.

5. World Health Organization: Global health observatory: raised blood pressure. Geneva; 2010
6. He J, Gu D, Chen J, Wu X, Kelly TN, Huang JF, Chen JC, Chen CS, Bazzano LA, Reynolds K, et al. Premature deaths attributable to blood pressure in China: a prospective cohort study. Lancet. 2009;374:1765-72.

7. Guo F, He D, Zhang W, Walton RG. Trends in prevalence, awareness, management, and control of hypertension among United States adults, 1999 to 2010. J Am Coll Cardiol. 2012;60:599-606.

8. Joffres M, Falaschetti E, Gillespie C, Robitaille C, Loustalot F, Poulter N, McAlister FA, Johansen H, Baclic O, Campbell N. Hypertension prevalence, awareness, treatment and control in national surveys from England, the USA and Canada, and correlation with stroke and ischaemic heart disease mortality: a cross-sectional study. BMJ Open. 2013;3:e003423.

9. Wu Y, Huxley R, Li L, Anna V, Xie G, Yao C, Woodward M, Li X, Chalmers J, Gao $R$, et al. Prevalence, awareness, treatment, and control of hypertension in China: data from the China National Nutrition and Health Survey 2002. Circulation. 2008;118:2679-86.

10. Liu Q, Wang B, Kong Y, Cheng KK. China's primary health-care reform. Lancet. 2011:377:2064-6.

11. Li H, Wei X, Wong MC, Yang N, Wong SY, Lao X, Griffiths SM. A comparison of the quality of hypertension management in primary care between Shanghai and Shenzhen: a cohort study of 3196 patients. Medicine (Baltimore). 2015:94:e455.

12. Wei X, Yang N, Gao Y, Wong SY, Wong MC, Wang J, Wang HH, Li DK, Tang J, Griffiths SM. Comparison of three models of ownership of community health centres in China: a qualitative study. J Health Serv Res Policy. 2015;20:162-9.

13. Kodner DL. All together now: a conceptual exploration of integrated care. Healthc Q. 2009:13 Spec No:6-15.

14. Sibiya MN, Gwele NS. A model for the integration of primary health-care services in the province of KwaZulu-Natal, South Africa. J Nurs Manag. 2013; 21:387-95.

15. Institute of Medicine. Primary care: American's health in a new era. Washington, D.C.: National Academy Press; 1996

16. Freeman G, Woloshynowych M, Baker R, Boulton M, Guthriem B, Car J, Haggerty J, Tarrant C. Continuity of care 2006: waht have we learned since 2000 and what are policy imperatives now? London: Report for the National Co-ordinating Center for NHS Service Delivery and Organization R\&D (NCCSDO); 2007.

17. McWhinney IR. Continuity of care in family practice. Part 2: implications of continuity. J Fam Pract. 1975;2:373-4.

18. Haggerty $\lrcorner$, Reid RJ, Freeman GK, Starfield BH, Adair CE, McKendry R. Continuity of care: a multidisciplinary review. BMJ. 2003;327:1219-21.

19. Nanjing Statistical Bureau. Yearbook of Nanjing statistics. 2013.

20. Wuhan Statistical Bureau. Yearbook of Wuhan statistics. 2013.

21. Shi L, Starfield B, Xu J, Politzer R, Regan J. Primary care quality: community health center and health maintenance organization. South Med J. 2003;96: $787-95$

22. Guthrie B. Continuity in UK general practice: a multilevel model of patient doctor and practice factors associated with patients seeing their usual doctor. Fam Pract. 2002;19:496-9.

23. Kristjansson E, Hogg W, Dahrouge S, Tuna M, Mayo-Bruinsma L, Gebremichael G. Predictors of relational continuity in primary care: patient, provider and practice factors. BMC Fam Pract. 2013;14:72.

24. Sweeney KG, Gray DP. Patients who do not receive continuity of care from their general practitioner-are they a vulnerable group? Br J Gen Pract. 1995:45:133-5.

25. Yang S, Wang S, Xiang X, Yang C, Chen H, Zhou Y, Liu G, Zhang X. Chines Hospital Manag. 2013:33:8-10.

26. Nanjing Health Bureau. The proposal on accelerating the establishment of health information system in Nanjing. 2013.

27. Rowan MS, Lawson B, MacLean C, Burge F. Upholding the principles of primary care in preceptors' practices. Fam Med. 2002;34:744-9.

28. Shi L, Lee DC, Liang $H$, Zhang L, Makinen M, Blanchet $N$, Kidane R, Lindelow M, Wang H, Wu S. Community health centers and primary care access and quality for chronically-ill patients - a case-comparison study of urban Guangdong Province. China Int J Equity Health. 2015;14:90.

29. Shi L, Makinen M, Lee DC, Kidane R, Blanchet N, Liang H, Li J, Lindelow M, Wang H, Xie S, Wu J. Integrated care delivery and health care seeking by chronically-ill patients - a case-control study of rural Henan province. China Int J Equity Health. 2015;14:98.

30. Wong MC, Wang HH, Wong SY, Wei X, Yang N, Zhang Z, Li H, Gao Y, Li DK, Tang J, et al. Performance comparison among the major healthcare financing systems in six cities of the Pearl River Delta region, mainland China. PLoS One. 2012;7:e46309. 
31. Wong SY, Kung K, Griffiths SM, Carthy T, Wong MC, Lo SV, Chung VC, Goggins WB, Starfield B. Comparison of primary care experiences among adults in general outpatient clinics and private general practice clinics in Hong Kong. BMC Public Health. 2010;10:397.

32. Sung NJ, Suh SY, Lee DW, Ahn HY, Choi YJ, Lee JH, Korean Primary Care Research G. Patient's assessment of primary care of medical institutions in South Korea by structural type. Int I Qual Health Care. 2010;22:493-9.

Submit your next manuscript to BioMed Central and we will help you at every step:

- We accept pre-submission inquiries

- Our selector tool helps you to find the most relevant journal

- We provide round the clock customer support

- Convenient online submission

- Thorough peer review

- Inclusion in PubMed and all major indexing services

- Maximum visibility for your research

Submit your manuscript at www.biomedcentral.com/submit
Biomed Central 